\title{
Improve Work Performance of Rice Whitening Machine by using New Design for Sieve
}

\author{
Sarmad Ahmed Abbas, Karim Hassan Ali and Fathi Abdel Sahib al Shamma \\ Department of Mechanical Engineering, University of Baghdad, Baghdad, Iraq \\ sarmdahh@gmail.com
}

\begin{abstract}
In this research, when designing the new model of the sieve, there are three important factors were introduced that affect the process of rice whitening and polishing. These factors are the length of the main shaft, the sieve diameter and the speed rotation of the main shaft. These three factors were introduced into the new design of the sieve through centrifugal force and its effect. The results showed that the practical side was identical with the theoretical side in the measurement of strain by stabilizing the strain gages at four angles $(0,10,45$ and 90$)$. In addition, the new design gave a whiting ratio of rice higher than the old design which was $32.9 \%$ while to old sieve give the white ratio is $29.7 \%$. And will also, prolong the life of mechanical parts of the machine better because the pregnancy will be less.
\end{abstract}

Key words: Sieve, factors, diameter, measurement, practical side, prolong

\section{INTRODUCTION}

Rice is one of the most important foods widely used in different parts of the world and is the second most consumed cereal in the Pacific Ocean and Asia. Where 60 of the world's population and 90 of the people of Asia and the Pacific are using their main food (FAO, 2013). He found that the high speed of the main shaft works to break down the rice, when the speed increases from $600-1000$, the rate of broken of rice increases. It was also, found that when the moisture is reduced to $13 \%$, rice broken is increased (Dilday, 1987; Roomi et al., 2004; Varnamkhasti et al., 2008). Developed the rice whiting machine was in Nigeria after its import was prevented using an oval sieve and the production rate of the machine was $1.5 \mathrm{t}$ per $8 \mathrm{~h}$ (Akintunde, 2007). Evolve the small rice grinding machine in order to support the agrarian community in Ubon Ratchathani Province and Chiang Mai Province by using the design of experiment technical. The result of a break ratio rice was $15.29 \%$ when the cylinder speed was $1560 \mathrm{rpm}$. In this case, we took three important factors and introduced them in the theoretical and practical part for the purpose of obtaining a machine with high bleaching quality and with long age.

Theoretical analysis: We divided the stress that occurs in the machine into two parts in the first section is bending the output of the centrifugal force. The second stress is produced from the shear force:

$$
\sigma=\frac{\operatorname{Mmax}^{*} \mathrm{y}}{1}
$$

where, $\mathrm{M}$ is the effect of centrifugal force along the length of the shaft (Khurmi and Gupta, 2005). And:

$$
\mathrm{y}=\frac{\mathrm{D}}{2}
$$

where, $\mathrm{D}$ is dameter of the shaft. And:

$$
\mathrm{I}=\frac{1}{2} * \mathrm{M}^{*} \mathrm{R}^{2}
$$

where, $\mathrm{R}$ is the radius of shaft:

$$
\tau=\mu \mathrm{F}_{\mathrm{N}} \mathrm{r}
$$

where, $\tau$ is shear force and $\mu$ is coefficient of friction between rice and serve. And $F_{N}$ is centrifugal force (Khurmi and Gupta, 2005):

$$
\mathrm{F}_{\mathrm{N}}=\mathrm{m}^{*} \omega^{2}{ }^{*} \mathrm{r}
$$

where, $\mathrm{m}$ is weighthe $\mathrm{t}$ of stone the $\mathrm{r}$ is the radius of stone. The $\omega$ is speed rotation of the motor. Of the two former sections can be extracted the max stress occurs on the sieve and on the shaft:

Corresponding Author: Sarmad Ahmed Abbas, Department of Mechanical Engineering, University of Baghdad, Baghdad, Iraq sarmdahh@gmail.com 


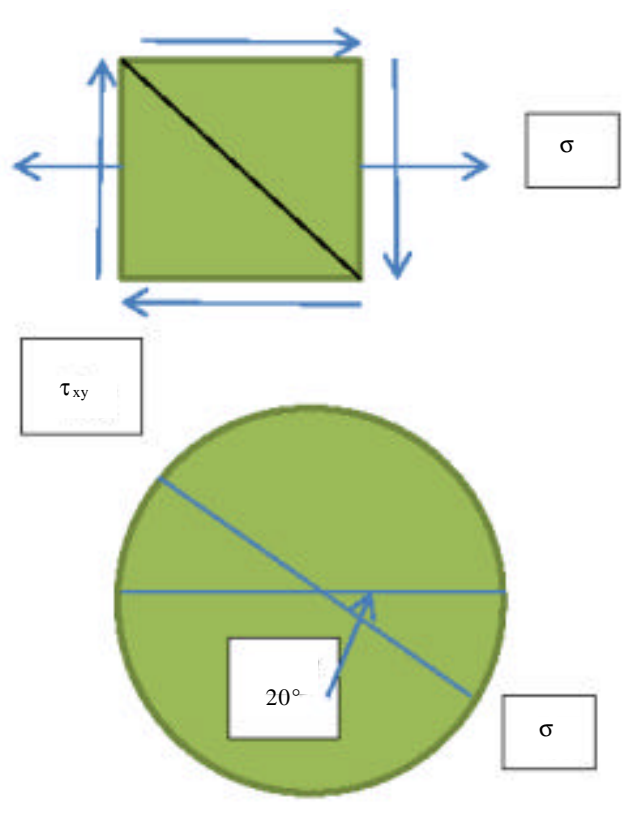

Fig. 1: The Mohr circle with the shear force and bending stress

$$
\begin{aligned}
& \sigma_{1}=\frac{1}{2} *\left(\frac{\mathrm{D}}{21}\right) *\left(\mathrm{M}+\sqrt{\mathrm{M}^{2}+\mathrm{T}^{2}}\right) \\
& \sigma_{2}=\frac{1}{2} *\left(\frac{\mathrm{D}}{21}\right) *\left(\mathrm{M}-\sqrt{\mathrm{M}^{2}+\mathrm{T}^{2}}\right)
\end{aligned}
$$

where, $\sigma_{1}$ and $\sigma_{2}$ are principle stress on shaft (Hearn, 1997). The max shear stress is:

$$
\begin{gathered}
\mathrm{T}_{\mathrm{e}}=\sqrt{\mathrm{M}^{2}+\mathrm{T}^{2}} \\
\tau_{\max }=\frac{\mathrm{T}_{\mathrm{e}} * \mathrm{D}}{2 \mathrm{~J}}
\end{gathered}
$$

where, $T_{e}$ is equivalent torqe (Hearn, 1997). The amount of power used by the machine is calculated by law:

$$
\mathrm{P}=\mathrm{T}_{\mathrm{e}} \omega
$$

The principle angle was calculated by drawing the Mohr circle from the shear force and bending stress (Khurmi and Gupta, 2005) (Fig. 1).

\section{MATERIALS AND METHODS}

Experimental analysis; The prototype of a whiting machine: This model is working at power $0.37 \mathrm{~kW}$

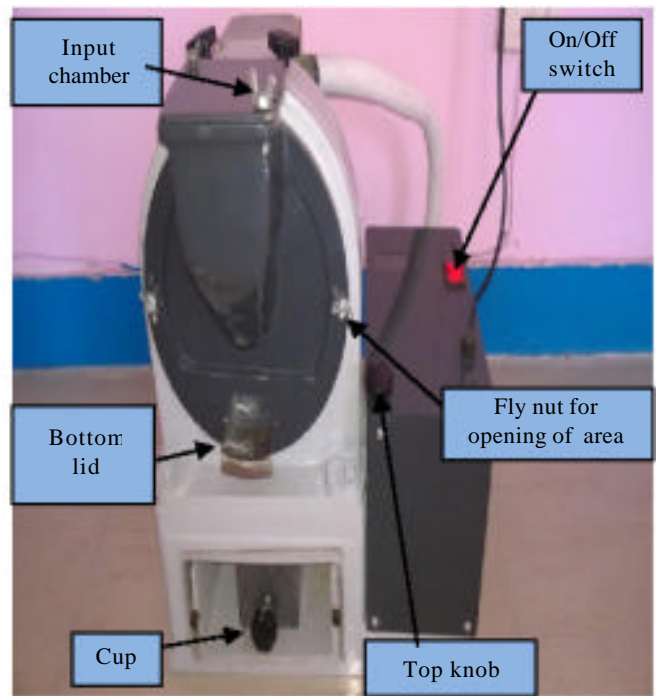

Fig. 2: The prototype of a whiting machine

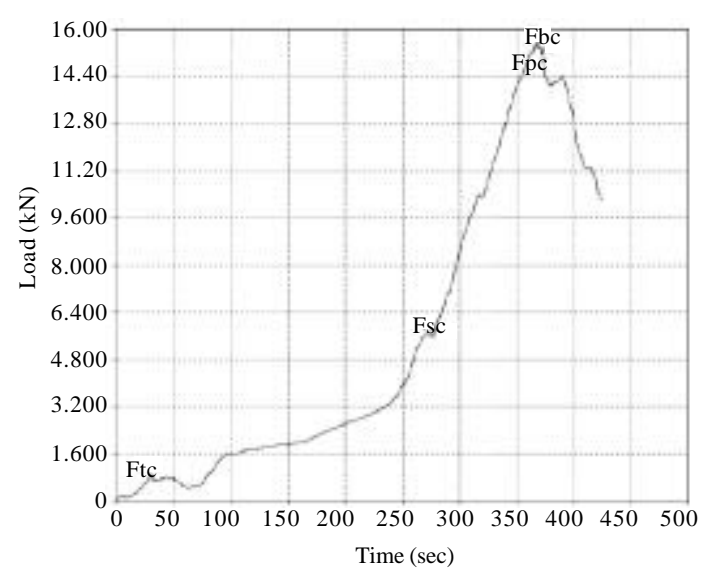

Fig. 3: The compression test for stone were the y-axis shows how much load is cast on the stone until the failure and $\mathrm{x}$-axis shows the time of stone failed

equivalent to $(1 / 2 \mathrm{HP})$ with a production capacity of $200 \mathrm{~g}$ every two min. In this type, the electric motor is connected directly to the main shaft. Through theoretical analysis has calculated the amount of energy needed by rice for bleaching (Fig. 2).

Stone abrasive: The mixture of milling cylinder consists of calcined mangnesite, magnesium chloride solution, emery and silicon carbide. The stone diameter is $15 \mathrm{~cm}$. The stone hardness was tested by the (laryee testing machine) To find out the duration of bearing the stone during the compression process (Fig. 3). Through the manufacture of 


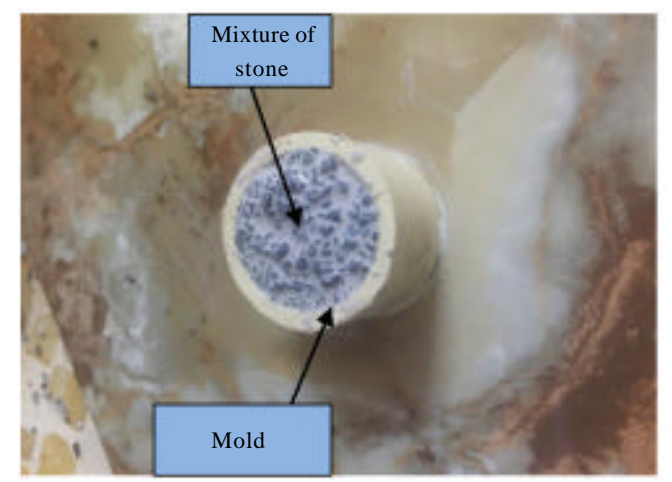

Fig. 4: The moulds of stone

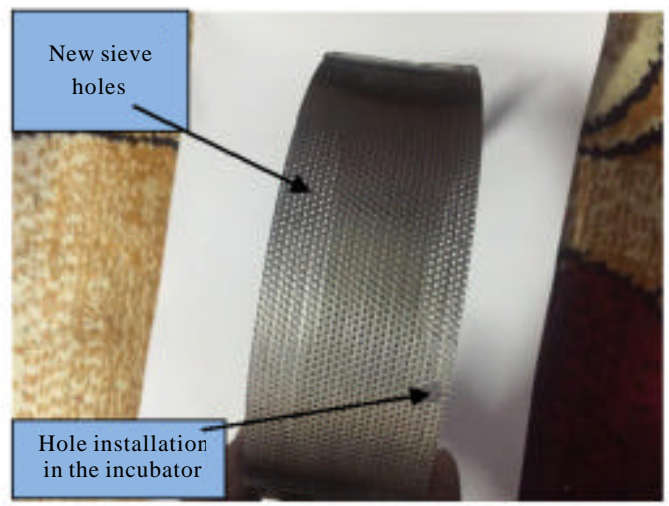

Fig. 5: The shape of the new holes for the designer sieve

moulds, especially, for a diameter of $3 \mathrm{~cm}$ and thickness of $2 \mathrm{~cm}$ and put the mixture of stone in the moulds, for a period 7 days (Fig. 4).

Figure 3 shows the compression test for stone were The $y$-axis shows how much load is cast on the stone until the failure and $\mathrm{x}$-axis shows the time of stone failed.

Designer sieve: The new sieve is designed to in a way that increases the surface area. The shape of the holes was changed from longitudinal to circular and $1 \mathrm{~mm}$ diameter. We also, reduced the number of holes to increase the surface area of bleaching (Fig. 5 and 6).

Strain gage: We have installed strain gage at four different angles. Where this angle was extracted through the theoretical side. This angle is $\left(0^{\circ}, 10^{\circ}, 45^{\circ}, 90^{\circ}\right)$ where the strain gage installs in this direction (Fig. 7). It is connected to the (data $\log$ ) device. Where strain is measured. It is a device used to measure strain and consists of eight channels and is characterized by measuring the strain very accurately and can read the stress in any direction (Fig. 8).

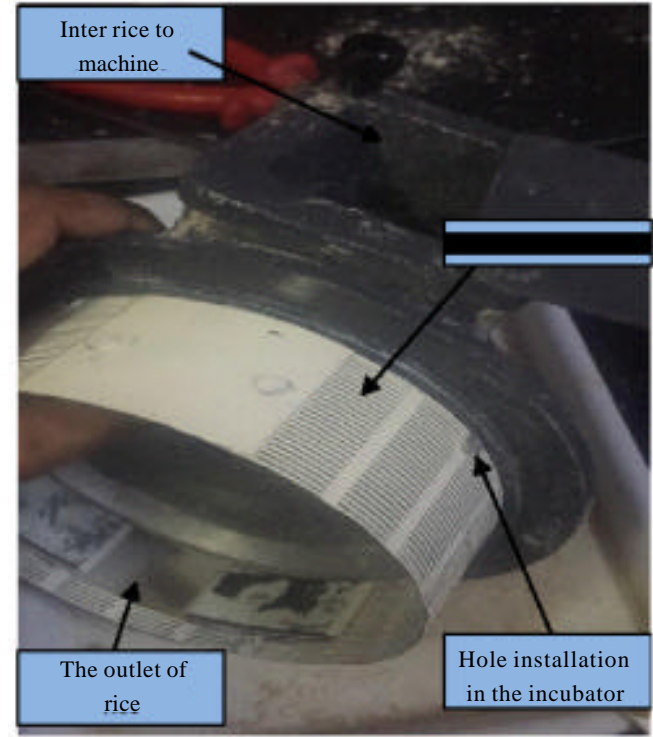

Fig. 6: The old user's sieve in the grinding process

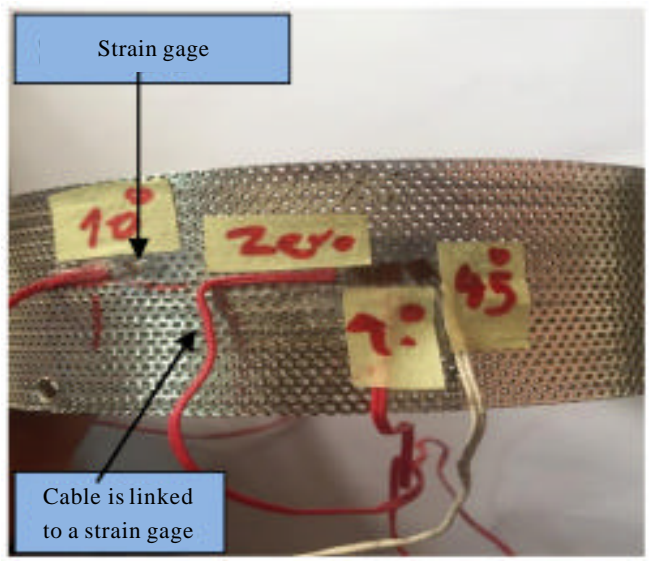

Fig. 7: The angle that was installed strain gage

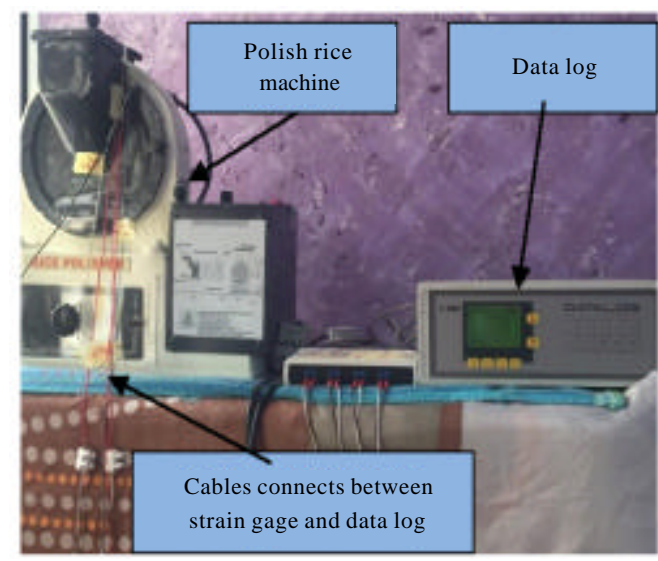

Fig. 8: The data log and polishing rice 


\section{RESULTS AND DISCUSSION}

A specific range has been determined that should not exceed at the design of any sieve and this range is the result of the bending stress divided on shear forces. If this range increases the decision, to cause the grinding of rice and if less than this range causes the broken of rice because of increasing in bending that lead to increase in to force. Speed is also an important role. In this model, we have speeded up more than $500 \mathrm{rpm}$ leading to grinding the rice and also when we reduced the speed less from 500 leading to broken the rice.

\section{CONCLUSION}

There are three factors that control the work of rice bleaching in the machine of whiting which is the length, diameter and speed. These three factors were introduced into the new design of the sieve through centrifugal force and its effect. The results showed that the practical side was identical with the theoretical side in the measurement of strain by stabilizing the strain gages at four angles $(0,10,45$ and 90). In addition, the new design gave a whiting ratio of rice higher than the old design which was $32.9 \%$ while to old sieve give the white ratio is $29.7 \%$. And will also prolong the life of mechanical parts of the machine better because the pregnancy will be less.

\section{REFERENCES}

Akintunde, M.A., 2007. Development of a rice polishing machine. AU J. T, 11: 105-112.

Dilday, R.H., 1987. Influence of thresher cylinder speed and grain moisture at harvest on milling yield of rice. Proc. Arkansas Acad. Sci., 41: 35-37.

FAO, 2013. FAO Statistical Yearbook 2013: World Food and Agriculture. Food and Agriculture Organization, Rome, Italy, ISBN-13: 9789251073964, Pages: 356.

Hearn, E.J., 1997. Mechanics of Materials 1: An Introduction to the Mechanics of Elastic and Plastic Deformation of Solids and Structural Materials. 3rd Edn., Butterworth-Heinemann, Oxford, England, UK.,.

Khurmi, R.S., J.K. Gupta, 2005. Theory of Machines. 14th Edn., S. Chand Group, New Dehli, India, ISBN-13:9788121925242, Pages: 1071.

Roomi, M.S.M., D.P. Senanayake and K.B. Palipana, 2004. Evaluation of milling performance with different types of rice polishing machines available in Sri Lanka. J. Eng., 1: 229-235.

Varnamkhasti, M.G., H. Mobli, A. Jafari, M.H. Soltanabadi and S.H. Rafiee, 2008. Investigation of rice quality of Sorkheh variety using a rotor equipped with screw conveyor in blade whitener. J. Sci. Technol. Agric. Nat. Resour., 12: 257-266. 